\title{
Rigidity of area minimizing tori in 3-manifolds of nonnegative scalar curvature
}

\author{
Mingliang Cai and Gregory J. Galloway ${ }^{1}$
}

The following conjecture arises from remarks in Fischer-Colbrie-Schoen ([FCS], Remark 4, p. 207): If $(M, g)$ is a complete Riemannian 3-manifold with nonnegative scalar curvature and if $\Sigma$ is a two-sided torus in $M$ which is suitably of least area then $M$ is flat. Such a result, as Fischer-Colbrie and Schoen commented, would be an interesting analogue of the CheegerGromoll splitting theorem. Here we present a proof of this conjecture assuming $\Sigma$ is of least area in its isotopy class. The proof is a consequence of the following local result, which is the main result of the paper.

Theorem 1. Let $(M, g)$ be a $C^{\infty} 3$-manifold with nonnegative scalar curvature, $S \geq 0$. If $\Sigma$ is a two-sided torus in $M$ which is locally of least area (see Section 2), then $M$ is flat in a neighborhood of $\Sigma$.

It follows that $\Sigma$ is flat and totally geodesic and that locally $M$ splits along $\Sigma$. A partial infinitesimal version of Theorem 1 was observed in [FCS], namely, if $\Sigma$ is a stable minimal two-sided torus in $M$ with nonnegative scalar curvature then $\Sigma$ must be flat and totally geodesic, and the scalar curvature and normal Ricci curvature of $M$ vanishes along $\Sigma$. In [CG] the authors proved Theorem 1 under the assumption that $M$ is analytic. The result in the analytic case follows as an immediate consequence of a more general result which holds for $C^{\infty}$ manifolds, see Theorem B in [CG]. Here we will make use of Theorem B to present a proof of Theorem 1.

We note that, under the assumptions of Theorem $1, M$ need not be globally flat. Consider, for example, $S^{1} \times S^{2}$, where $S^{2}$ is a sphere which is flattened near the equator $E$. Then $S^{1} \times E$ is a torus which is locally of least area in $S^{1} \times S^{2}$.

The idea of the proof of Theorem 1 is as follows. It is first shown that $\Sigma$ cannot be locally strictly of least area. If it were, then under a sufficiently small perturbation of the metric to a metric of (strictly) positive scalar

\footnotetext{
${ }^{1}$ This work has been partially supported by NSF grant DMS-9803566.
} 
curvature, $\Sigma$ would be perturbed to a torus which is still locally of least area. But this would contradict the fact that a compact two-sided stable minimal surface in a 3-manifold with strictly positive scalar curvature must be a sphere, cf. Theorem 5.1 in [SY1]. It is then shown that on each side of $\Sigma$ there is a torus which is locally of least area. By cutting out the region bounded by these two tori and pasting it appropriately to a second copy, one obtains, using Theorem B, a smooth 3-torus with nonnegative scalar curvature. By Schoen and Yau [SY], this 3-torus must be flat, and Theorem 1 follows. We now proceed to a detailed proof of Theorem 1 .

In all that follows we work in the $C^{\infty}$ category. For simplicity, all surfaces are assumed to be embedded. However, by pulling back to the normal bundle of $\Sigma$, it is clear that a version of Theorem 1 holds for immersed surfaces, as well. By definition, a compact two-sided surface $\Sigma$ in a 3-manifold $M$ is locally of least area provided in some normal neighborhood $V$ of $\Sigma, A(\Sigma) \leq$ $A\left(\Sigma^{\prime}\right)$ for all $\Sigma^{\prime}$ isotopic to $\Sigma$ in $V$, where $A$ is the area functional. If the inequality is strict for $\Sigma^{\prime} \neq \Sigma$, we say that $\Sigma$ is locally strictly of least area. Note that "locally of least area" implies "stable minimal".

Let $V$ be a normal neighborhood of a compact two-sided surface $\Sigma$ in a 3 -manifold $M$. Then, via the normal exponential map, $V=(-\ell, \ell) \times \Sigma$, and the metric $g=d s^{2}$ takes the form,

$$
d s^{2}=d t^{2}+\sum_{i, j=1}^{2} g_{i j}(t, x) d x^{i} d x^{j} .
$$

The following is a restatement of part of Theorem B in [CG].

Lemma 1. Let $(M, g)$ be a 3-manifold with nonnegative scalar curvature, $S \geq 0$. Suppose $\Sigma$ is a two-sided torus in $M$ which is locally of least area. Then with respect to geodesic normal coordinates along $\Sigma$ (see equation 1),

$$
\frac{\partial^{n} g_{i j}}{\partial t^{n}}(0, x)=0
$$

for all $n$ and all $x \in \Sigma$.

Lemma 1 is used below to ensure that after certain cut and paste operations the resulting metric is smooth.

Lemma 2. Suppose $\Sigma$ is a compact two-sided surface in a 3-manifold $(M, g)$ with nonnegative scalar curvature, $S(g) \geq 0$. Then there exists a neighborhood $U$ of $\Sigma$ and a sequence of metrics $\left\{g_{n}\right\}$ on $U$ such that $g_{n} \rightarrow g$ in $C^{\infty}$ topology on $U$, and each $g_{n}$ has strictly positive scalar curvature, $S\left(g_{n}\right)>0$. 
Proof. Let $V=(-\ell, \ell) \times \Sigma$ be a normal neighborhood of $\Sigma$, so that the metric $g$ takes the form (1). Consider the sequence of metrics $g_{n}=e^{-2 n^{-1} t^{2}} g$. A straight forward computation gives,

$$
S\left(g_{n}\right)=e^{2 n^{-1} t^{2}}\left(S(g)+8 n^{-1}\left(1+t H_{t}-n^{-1} t^{2}\right)\right),
$$

where $H_{t}$ is the mean curvature (in the metric $g$ ) of $\Sigma_{t}=\{t\} \times \Sigma$. It is clear that by taking $\ell$ sufficiently small and $n$ sufficiently large we have $S\left(g_{n}\right)>0$.

In the next lemma we show that if $\Sigma \subset M$ is locally strictly of least area then by perturbing the metric of $M$ slightly, $\Sigma$ gets perturbed to a surface which is still locally of least area.

Lemma 3. Suppose $\Sigma$ is a compact two-sided surface in $\left(M^{3}, g\right)$ which is locally strictly of least area. Let $\left\{g_{n}\right\}$ be any sequence of metrics such that $g_{n} \rightarrow g$ in $C^{\infty}$ topology. Then for any neighborhood $U$ of $\Sigma$ and any positive integer $N$ there exists, for some $n \geq N$, a surface $\Sigma_{n} \subset U$ isotopic to $\Sigma$ in $U$ which is locally of least area in $\left(M, g_{n}\right)$.

Proof. The proof makes use of basic existence and convergence results for least area surfaces. Let $V=[-\ell, \ell] \times \Sigma$ be a compact normal neighborhood of $\Sigma$ contained in $U$, and restrict attention to the compact Riemannian manifold-with-boundary $(V, g)$. Since $\Sigma$ is locally strictly of least area, we can choose $\ell$ sufficiently small so that,

$$
A_{g}(\Sigma)<A_{g}\left(\Sigma^{\prime}\right) \quad \text { for all } \Sigma^{\prime} \in \mathcal{I}(\Sigma), \Sigma^{\prime} \neq \Sigma,
$$

where $\mathcal{I}(\Sigma)$ is the isotopy class of $\Sigma$ in $V$, and $A_{g}$ is the area functional in the $g$ metric.

Set $V_{0}=\left[-\frac{\ell}{2}, \frac{\ell}{2}\right] \times \Sigma$. Let $f=f(t)$ be a smooth nonnegative function on $[-\ell, \ell]$ such that $f=0$ on $\left[-\frac{\ell}{2}, \frac{\ell}{2}\right]$. By making the derivatives $f^{\prime}( \pm \ell)$ sufficiently large in absolute value, with $f^{\prime}(\ell)>0$ and $f^{\prime}(-\ell)<0$, we obtain a conformally related metric $\bar{g}=e^{f} g$ with the following properties.

(1) $\left.\bar{g}\right|_{V_{0}}=\left.g\right|_{V_{0}}$.

(2) $(V, \bar{g})$ has strictly mean convex boundary, i.e., $\partial V$ has positive mean curvature.

(3) For all $\Sigma^{\prime} \in \mathcal{I}(\Sigma)$ such that $\Sigma^{\prime} \neq \Sigma, A_{\bar{g}}(\Sigma)<A_{\bar{g}}\left(\Sigma^{\prime}\right)$. 
For each $n$, set $\bar{g}_{n}=e^{f} g_{n}$. Then the metrics $\bar{g}_{n}$ satisfy: (1) $\left.\bar{g}_{n}\right|_{V_{0}}=\left.g_{n}\right|_{V_{0}}$, (2) $\bar{g}_{n} \rightarrow \bar{g}$ in $C^{\infty}$ topology and (3) for $n$ sufficiently large, $\left(V, \bar{g}_{n}\right)$ has mean convex boundary. For each such $n$ let,

$$
\alpha_{n}=\inf _{\Sigma^{\prime} \in \mathcal{I}(\Sigma)} A_{\bar{g}_{n}}\left(\Sigma^{\prime}\right)
$$

Then by Theorem 5.1 and Section 6 in [HS] (see also [MSY]) there exists for each $n$ a surface $\Sigma_{n} \in \mathcal{I}(\Sigma)$ such that $A_{\bar{g}_{n}}\left(\Sigma_{n}\right)=\alpha_{n}$. In applying the results from [HS] we have used the fact that $V$ is $P^{2}$-irreducible (provided $\Sigma \neq S^{2}, P^{2}$ ) and that $V$ does not contain any compact one-sided surfaces. (If $\Sigma=S^{2}$ or $P^{2}$, one may appeal to Theorem 5.2 in [HS] and use specific features of the topology of $[-\ell, \ell] \times \Sigma)$.

For each $n, \Sigma_{n}$ is a compact stable minimal surface in $\left(V, \bar{g}_{n}\right)$, and the sequence $\left\{\alpha_{n}\right\}$ is bounded. It then follows by well-known convergence arguments that there is a subsequence of surfaces, call it again $\left\{\Sigma_{n}\right\}$, which converges locally in $C^{\infty}$ topology to a compact (embedded) minimal surface $\bar{\Sigma}$ in $(V, \bar{g})$; see especially Section 2.2 in [M], which applies fairly directly to the situation considered here. By the nature of the convergence, $\left\{\Sigma_{n}\right\}$ is eventually contained in any tubular neighborhood of $\bar{\Sigma}$, and for $n$ sufficiently large, $\Sigma_{n}$ will be transverse to the normal geodesics of $\bar{\Sigma}$. It follows that $\Sigma_{n}$ covers $\bar{\Sigma}$ via projection along the normal geodesics. Since $\bar{\Sigma}$ is necessarily two-sided (again, because $V$ does not contain any compact one-sided surfaces), it follows that the covering of $\bar{\Sigma}$ by $\Sigma_{n}$ must be one-sheeted, i.e., projection along the normal geodesics of $\bar{\Sigma}$ provides a diffeomorphism of $\Sigma_{n}$ onto $\bar{\Sigma}$; see e.g., $[\mathrm{S}]$.

Thus, $\bar{\Sigma}$ is isotopic to $\Sigma$ since each $\Sigma_{n}$ is. Furthermore, we have,

$$
A_{\bar{g}}(\bar{\Sigma})=\lim _{n \rightarrow \infty} \alpha_{n} \leq \lim _{n \rightarrow \infty} A_{\bar{g}_{n}}(\Sigma)=A_{\bar{g}}(\Sigma)
$$

Since $\Sigma$ is strictly of least area in its isotopy class in $(V, \bar{g})$, we conclude that $\bar{\Sigma}=\Sigma$. But by the above convergence, this means that for $n$ large enough, $\Sigma_{n}$ is contained in int $V_{0}$, in which $\bar{g}_{n}=g_{n}$. It follows that, for such $n, \Sigma_{n}$ is locally of least area in $\left(V, g_{n}\right)$. This concludes the proof of Lemma 3.

Proof of Theorem 1. Let $V=(-\ell, \ell) \times \Sigma$ be a normal neighborhood of $\Sigma$ with metric $g$ as in equation (1). Choose $\ell$ sufficiently small so that $\Sigma$ is of least area in its isotopy class in V. For technical reasons we modify the metric $g$ as follows. Let $\hat{g}$ be the metric on $V$ of the form (1) but with 
component functions $\hat{g}_{i j}, 1 \leq i, j \leq 2$, defined by,

$$
\hat{g}_{i j}(t, x)= \begin{cases}g_{i j}(t, x), & \text { for } t \in[0, \ell) \\ g_{i j}(-t, x), & \text { for } t \in(-\ell, 0] .\end{cases}
$$

$(V, \hat{g})$ is a smooth Riemannian manifold (by Lemma 1 ) such that $S(\hat{g}) \geq 0$ and reflection across $\Sigma,(t, x) \mapsto(-t, x)$, is an isometry. Further, $\Sigma$ is of least area in its isotopy class in $(V, \hat{g})$. By choosing $\ell$ even smaller if necessary, we guarantee that Lemma 2 holds for the neighborhood $U=V$.

If $\Sigma$ were strictly of least area in its isotopy class in $(V, \hat{g})$ then Lemmas 2 and 3 would imply that there is a two-sided stable minimal torus $\Sigma^{\prime}$ near $\Sigma$ with respect to some metric of strictly positive scalar curvature on $V$. This would contradict Theorem 5.1 in [SY]. Thus, there exists a surface $\bar{\Sigma} \in \mathcal{I}(\Sigma), \bar{\Sigma} \neq \Sigma$ such that $A_{\hat{g}}(\bar{\Sigma})=A_{\hat{g}}(\Sigma)$. Hence, $\bar{\Sigma}$ is also of least area in its isotopy class.

We claim that $\bar{\Sigma}$ is contained in one of the components of $V \backslash \Sigma$. If not, then $\bar{\Sigma}$ and $\Sigma$ must meet. Since $\bar{\Sigma}$ and $\Sigma$ are stable minimal tori in $(V, \hat{g})$ they must be totally geodesic (cf. [FCS]). Since they are totally geodesic and distinct, they must meet transversally. Thus, the intersection of $\bar{\Sigma}$ and $\Sigma$ will consist of a finite number of circles. By reflecting the portion of $\bar{\Sigma}$ in $(-\ell, 0] \times \Sigma$ across $\Sigma$ to $[0, \ell) \times \Sigma$ and smoothing out the resulting ridge along the circles of intersection, we obtain a surface isotopic to $\Sigma$ with less area than $\Sigma$, which is a contradiction. Thus, $\bar{\Sigma}$ lies to one side of $\Sigma$ and does not meet $\Sigma$.

These arguments imply that in the original Riemannian manifold $(V, g)$ there exist two tori $\Sigma^{+}$and $\Sigma^{-}$, one on each side of $\Sigma$, each isotopic to $\Sigma$ and each locally of least area. Let $W$ be the region in $V$ bounded by $\Sigma^{+}$ and $\Sigma^{-}$. Standard properties of isotopies $[\mathrm{H}]$ imply that $W$ has topology $[-1,1] \times T^{2}$. By taking two copies of $W$ and gluing them appropriately along their boundaries, we obtain, by Lemma 1, a smooth Riemannian manifold with nonnegative scalar curvature which is diffeomorphic to a 3-torus. By Schoen and Yau [SY], this 3-torus, and hence $W$ must be flat.

By fairly standard continuation arguments, Theorem 1 can be globalized as follows.

Theorem 2. Let $M$ be a complete connected 3-manifold of nonnegative scalar curvature whose boundary (possibly empty) is mean convex. If $M$ contains a two-sided torus $\Sigma$ which is of least area in its isotopy class then $M$ is flat. 
Proof. By the maximum principle, either $\Sigma$ is a boundary component of $M$ or $\Sigma$ is in the interior of $M$. If $\Sigma$ is a boundary component, let $M_{0}=M$. If $\Sigma$ is in the interior and disconnects $M$, let $M_{0}=\bar{U}_{0}$, where $U_{0}$ is one of the components of $M \backslash \Sigma$. If $\Sigma$ is in the interior and does not disconnect $M$, let $M_{0}$ be the manifold with boundary obtained by "separating" $M$ along $\Sigma$. In all cases, $\Sigma$ is a boundary component of $M_{0}$. To prove Theorem 2 it suffices to show that $M_{0}$ is flat. Consider the normal exponential map $\Phi:[0, \infty) \times \Sigma \rightarrow M_{0}$ along $\Sigma$ defined by $\Phi(t, x)=\exp _{x} t N$, where $\mathrm{N}$ is the inward pointing unit normal along $\Sigma$. (Note $\Phi$ need not be defined on all of $[0, \infty) \times \Sigma$.)

By Theorem 1, $M_{0}$ is flat in a neighborhood of $\Sigma$. (It is easily seen that Theorem 1 is still valid if $\Sigma$ is a boundary component.) Then, by standard arguments (which require only nonnegative Ricci curvature), since $\Sigma$ is locally of least area there exists $a>0$ such that $\Phi:[0, a) \times \Sigma \rightarrow$ $\Phi([0, a) \times \Sigma)$ is an isometry. (Here [0,a) $\times \Sigma$ carries the standard product metric and hence is flat since $\Sigma$ is). Let $\ell$ be the largest number (possibly $\infty)$ such that $\Phi:[0, \ell) \times \Sigma \rightarrow \Phi([0, \ell) \times \Sigma)$ is an isometry. Consider first the case $\ell=\infty$. Using that the limit of a sequence of normal geodesics to $\Sigma$ is a normal geodesic to $\Sigma$, one easily verifies that $\Phi([0, \infty) \times \Sigma)$ is both open and closed in $M_{0}$. Hence, $\Phi([0, \infty) \times \Sigma)=M_{0}$ and $M_{0}$ is flat.

Now consider the case $\ell<\infty$. Since $M_{0}$ is complete, each normal geodesic to $\Sigma, \gamma_{x}: t \mapsto \Phi(t, x), 0 \leq t<\ell$, extends to $t=\ell$. Suppose that $\Phi:[0, \ell] \times \Sigma \rightarrow \Phi([0, \ell] \times \Sigma)$ is an isometry. Then $\Sigma_{\ell}=\Phi(\{\ell\} \times \Sigma)$ is an embedded totally geodesic torus in $M_{0}$ which is locally of least area. By the maximality of $\ell, \Sigma_{\ell}$ must meet some component $\Sigma^{\prime}$ of $\partial M_{0}$. By the maximum principle for hypersurfaces, $\Sigma_{\ell}$ and $\Sigma^{\prime}$ must agree, $\Sigma_{\ell}=\Sigma^{\prime}$. One can now argue that $\Phi([0, \ell] \times \Sigma)$ is both open and closed in $M_{0}$. Hence, $M_{0}=\Phi([0, \ell] \times \Sigma)$ is flat.

Now suppose $\Phi:[0, \ell] \times \Sigma \rightarrow \Phi([0, \ell] \times \Sigma)$ is not an isometry. The only way this can happen is if two normal geodesics to $\Sigma, \gamma_{x_{i}}, i=1,2$, meet at $t=\ell, \gamma_{x_{1}}(\ell)=\gamma_{x_{2}}(\ell)$. Since there can be no focal points to $\Sigma$ along $\left.\gamma_{x_{i}}\right|_{[0, \ell]}$, there exists a neighborhood $U_{i}$ of $x_{i}$ in $\Sigma$ such that $\Phi:[0, \ell] \times U_{i} \rightarrow$ $\Phi\left([0, \ell] \times U_{i}\right)$ is an isometry. Hence, $\Phi\left(\{\ell\} \times U_{i}\right)$ is an embedded totally geodesic hypersurface in $M_{0}$ which (by the choice of $\ell$ ) is a constant distance $\ell$ from $\Sigma$. It follows that $\Phi\left(\{\ell\} \times U_{1}\right)$ and $\Phi\left(\{\ell\} \times U_{2}\right)$ must agree near the common end point $\gamma_{x_{1}}(\ell)=\gamma_{x_{2}}(\ell)$. By a straight forward continuation argument we conclude that each geodesic segment $\gamma_{x}, x \in \Sigma$, of length $2 \ell$ meets $\Sigma$ orthogonally at both end points. It is now easily argued that $\Phi([0, \ell] \times \Sigma)$ is both open and closed in $M_{0}$. Hence, $M_{0}=\Phi([0, \ell] \times \Sigma)$ is flat, and the proof of Theorem 2 is complete. 
We make some concluding remarks.

1. The results presented here were motivated in part by certain problems concerning the topology of black holes in general relativity, cf., [CG] [G1], [G2].

2. The example mentioned after Theorem $1, M=S^{1} \times S^{2}$, with $S^{2}$ flattened near the equator $E$, and $\Sigma=S^{1} \times E$, shows that stability is not sufficient to imply flatness. Assume the $S^{1}$ factor and $E$ have the same radius. Cutting $M$ along $\Sigma$ we obtain two solid tori, the boundary of each of which is a copy of $\Sigma$. Gluing the two solid tori back together along their toroidal boundaries after a suitable twist we obtain a manifold $M^{\prime}$ diffeomorphic to $S^{3}$ with nonnegative scalar curvature which contains a stable minimal torus. Applying Theorem A in [GL], which is proved by a local construction, we can add an asymptotically flat end to $M^{\prime}$ to obtain an asymptotically flat manifold diffeomorphic to $\mathbb{R}^{3}$ with nonegative scalar curvature which contains a stable minimal torus. In the language of general relativity, we have obtained an asymptotically flat time symmetric initial data set on $\mathbb{R}^{3}$ satisfying the constraint equations which contains a stable toroidal apparent horizon. However, we know of no such vacuum (scalar flat) examples, and conjecture that there are none.

3. Using the higher dimensional work of Schoen and Yau [SY2] it appears that Theorem 1 can be extended to higher dimensions as follows: Let $M^{n}$ have nonnegative scalar curvature. If $\Sigma$ is a compact two-sided hypersurface in $M^{n}$ which does not admit a metric of positive scalar curvature and which is locally of least area then a neighborhood of $\Sigma$ splits. We are grateful to a referee for suggesting an alterative proof of Lemma 3, valid in higher dimensions, which makes this generalization possible. Further aspects of this will be discussed elsewhere.

4. In [FCS], Fischer-Colbrie and Schoen proved that a complete stable minimal surface in an orientable 3-manifold with nonnegative scalar curvature must be conformal to the complex plane or the cylinder $A$. In the latter case it has been shown that $A$ is flat and totally geodesic, cf., [FCS] and [CM]. The example $M=\mathbb{R} \times S^{2}$, where $S^{2}$ is flattened near the equator, shows that $M$ need not be flat. However, in view of the results cited and the results presented here, it seems reasonable to conjecture that if the cylinder $A$ is actually area minimizing (in a suitable sense) then $M$ is flat (cf., Remark 4 in [FCS]). 
We would like to express our gratitude to a referee and an anonymous reviewer for many valuable comments and suggestions. We would also like to thank Tobias Colding for some helpful discussions.

\section{References.}

[CG] M. Cai and G.J. Galloway, Least area tori and 3-manifolds of nonnegative scalar curvature, Math. Z. 223 (1996), 387-395.

[CM] T.H. Colding and W.P. Minicozzi, Estimates for parametric elliptic integrals, Preprint, 1999.

[FCS] D. Fischer-Colbrie and R. Schoen, The structure of complete stable minimal surfaces in 3-manifolds of nonnegative scalar curvature, Commun. Pure Applied Math. 33 (1980), 199-211.

[G1] G.J. Galloway, On the topology of black holes, Commun. Math. Phys. 151 (1993), 53-66.

[G2] Least area tori, black holes and topological censorship, Contemp. Math. Proceedings of Special Session on Mathematical Physics and Differential Geometry, ed. J. K. Beem and K. L. Duggal, 170 (1994), 113123.

[GL] M. Gromov and H.B. Lawson, Jr., The classification of simply connected manifolds of positive scalar curvature, Ann. of Math. 111 (1980), 423-434.

[H] M.W. Hirsch, Differential topology, Springer-Verlag, New York 1976.

[HS] J. Hass and P. Scott, The existence of least area surfaces in 3-manifolds, Trans. Amer. Math. Soc. 310 (1988), 87-114.

[S] P. Scott, On sufficiently large 3-manifolds, Quart. J. Math. Oxford, 23 (1972), 159-172.

[M] A.K.M. Masood-ul-Alam, Ph. D. Thesis, Australian National University, Canberra 1987.

[MSY] W. Meeks, L. Simon and S.-T. Yau, Embedded minimal surfaces, exotic spheres, and manifolds with positive Ricci curvature, Ann. Math. 116 (1982), 621-659.

[SY1] R. Schoen and S.-T. Yau, Existence of incompressible minimal surfaces and the topology of three dimensional manifolds with nonnegative scalar curvature, Ann. Math. 110 (1979), 127-142.

[SY2] _ On the structure of manifolds with positive scalar curvature, Manuscripta Math. 28 (1979), 159-183. 
UNIVERSITY OF MIAMI

Coral Gables, FL 33124

Received May 8, 1998. 